\title{
26
}

\section{Can Equivalent Capacity CAC Deal with Worst Case Traffic in GCRA-Policed ATM Networks?}

Kai Schmidt, Maike Wichers, Ulrich Killat

University of Technology Hamburg-Harburg

Digital Communication Systems

21071 Hamburg, Germany,

Phone+49-40-7718-3049, email: killat@tu-harburg.de

\begin{abstract}
In order to manage modern ATM networks various methods for connection admission control (CAC) have been proposed. In addition, usage parameter control (UPC) algorithms supervise the transmitted traffic to prevent the user from exceeding the negotiated traffic parameters. This paper addresses the question, whether the broadly accepted Equivalent Capacity CAC algorithm can guarantee the quality of service asserted to the subscribers provided that the algorithm has only knowledge of the specified GCRA parameters used for UPC. We describe two types of worst case traffic conforming to the Generic Cell Rate Algorithm and show the performance of Equivalent Capacity CAC in various traffic scenarios.
\end{abstract}

Keywords

ATM Network, CAC, UPC, GCRA, Worst Case Traffic, Simulation

\section{INTRODUCTION}

Since the Asynchronous Transfer Mode (ATM) has been proposed for the upcoming Broadband ISDN by the ITU-T, a considerable number of methods for connection admission control (CAC) have been scrutinised. Reviews like $[4,5,6,7]$ provide a comprehensive overview. Each CAC algorithm requires a CAC specific set of traffic parameters describing the statistical properties of the cell streams to be dealt with by the CAC. Based on these traffic parameters a traffic contract is made if resources are available to accommodate the requested connection. In order to protect the network from overload and to guarantee the quality of already established connections it is necessary to control whether the admitted cell streams meet their respective traffic contracts. The usage parameter control (UPC) identifies cells of a certain cell stream as being conforming or non-conforming to the traffic contract. An in- 
tricate problem is that the UPC in general will not operate on the same traffic parameters as the CAC does. Therefore, a sometimes ambiguous translation of the CAC traffic parameter set to a set of UPC traffic parameters becomes necessary.

The key idea pursued in this paper is the following:

The network must be prepared for the situation in which the user tries to transmit a maximum of data conforming to the specified UPC parameters. Now, this can be done by submitting a constant rate cell stream or as another extreme by submitting bursty traffic streams. In this context worst case behaviour turns out to be periodic bursty traffic with a maximum admissible burst size.

In this paper we will identify two types of worst case traffic which are described by a set of GCRA [1] parameters. These traffic streams that are fully conforming to their respective GCRAs have two remarkable properties: They to show worst case behaviour with respect to buffer demands and their parameter set can unambiguously be translated to an Equivalent Capacity [3] parameter set.

In [2] B. T. Doshi shows in a qualitative analysis that for certain traffic streams the Equivalent Capacity CAC will be too optimistic. We will address the question whether the combination of connection admission control based on the Equivalent Capacity Algorithm and usage parameter control based on the GCRA will be able to protect the network from overload and evaluate the performance of the Equivalent Capacity Algorithm in a realistic network environment.

\section{WORST CASE TRAFFIC}

Having specified an algorithm for usage parameter control - the Generic Cell Rate Algorithm - the question arises, how a worst case cell stream could look like. From the network operators point of view worst case traffic will be most demanding according to the buffer occupancy inside a multiplexor. We will assume a single ATM multiplexor having a number of $N$ input lines. The two types of worst case traffic which we will describe in the following will be determined by a given set of GCRA parameters so that all cells are conforming to this parameter set. Thereby, the cell streams are constructed in such a way that the superposition of these traffic streams will require a maximum number of buffers within the multiplexor.

Following the ATM-Forum Traffic Management Specification [1] we consider the four parameter $\operatorname{GCRA}\left(T, \tau, T_{s}, \tau_{s}\right)$ equivalent to a two stage leaky bucket. GCRA $\left(T_{s}, \tau_{s}\right)$ representing the second leaky bucket controls the mean cell rate where $T_{s}$ and $\tau_{s}$ correspond to the buckets leak and size, respectively. Mutatis mutandis the $\operatorname{GCRA}(T, \tau)$ is applied for peak cell rate policing. Since we do not want the peak cell rate to be exceeded $\tau=0$ (Peak Rate Enforcement). 


\subsection{Worst Case Traffic A - Greedy ON/OFF}

Intuitively the worst case traffic should be a traffic where leaky bucket equivalent to the GCRA is filled with a burst of cells emitted at peak cell rate followed by a silence period until the leaky bucket is again empty. Then the next burst will follow. We will call a source emitting this type of worst case traffic as greedy ON/OFF source emitting worst case traffic type $A(W C T-A)$.

As an example in Figure 1 the worst case traffic is described by the GCRA parameter set $\left(T, \tau, T_{s}, \tau_{s}\right)=(2,0,8,23)$. The peak cell rate of each source is $50 \%$, the mean cell rate $12.5 \%$, and the maximum burst size (=mean burst size) is 4 cells according to equation (7). The cell stream is periodic with a period of $\ell_{A}=\left\lceil M B S \cdot T_{s}\right\rceil$ because the GCRA requires $T_{s}$ time slots for each cell in the peak cell rate burst to recover.

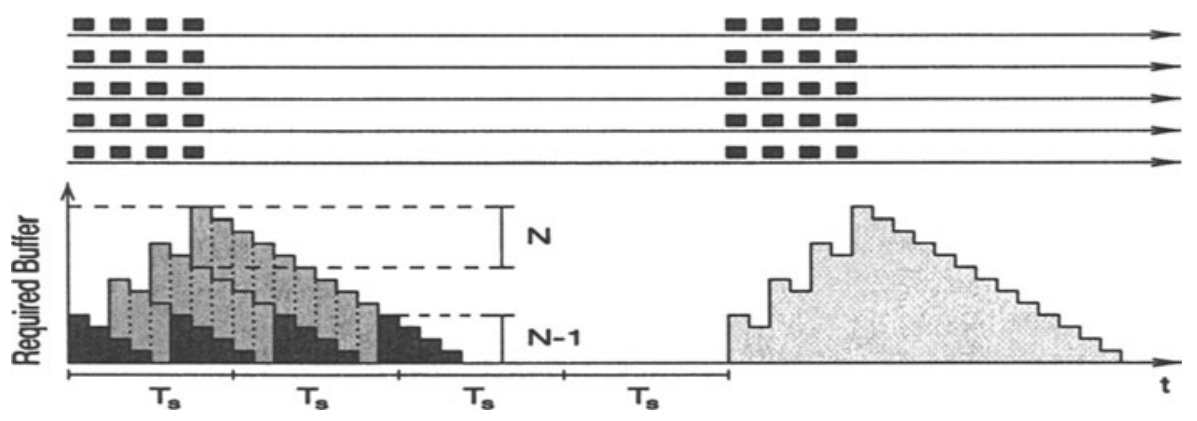

Figure 1 Synchronous Greedy ON/OFF Streams

In Figure 1 the buffer occupancy in the switch is shown, when $N=5$ lines of synchronised worst case traffic streams are superposed in a multiplexer. The necessary buffer size $K_{A}=13$ can be calculated directly from the number of cell streams $N$ and the worst case traffic parameter set:

$K_{A}=M B S \cdot N-\lceil(M B S-1) \cdot T\rceil-1$

Even if the buffer size is sufficient for the superposition of the cell streams, it is important to know the remaining number of buffers for additional traffic provided the minimum buffer size $K_{A}$ has been implemented. Figure 1 shows a graphical approach to calculate the number of occupied buffers for the greedy ON/OFF traffic. Parting the graphic in two areas (light grey and dark grey) makes it easy to derive an analytical expression for the mean number of free buffers. The total amount of available buffer during one period is determined by the period length $\ell_{A}$ and buffer size $K_{A}$. For a given value of $K_{A}$ the mean number of free buffers $\kappa_{A}$ is 
$\kappa_{A}=K_{A}-\frac{1}{\ell_{A}}\left\{\frac{M B S \cdot N}{2}[M B S(N-T)+T-1]\right\}$ cells/time slot

The smaller the mean number of free buffers is, the more likely cells will be lost if additional traffic is passing the buffer. We will later on use $\kappa$ to determine how much "worst case" a traffic stream is.

\subsection{Worst Case Traffic Type B}

The greedy ON/OFF traffic described above fully exploits the amount of traffic the UPC allows. However, the greedy ON/OFF traffic is not the only type of traffic that transmits the maximum number of cells during a period of time. We will present the worst case traffic type B as such a type of traffic, and we will show that this type of traffic can be more demanding with respect to both the necessary buffer size and the mean number of occupied buffers per time slot.

Similar to the greedy ON/OFF traffic the empty leaky bucket is filled with a burst at peak cell rate but now the peak rate burst is followed by the emission of one cell at the earliest possible time slot.

To understand why this traffic behaviour is worse than the greedy ON/OFF traffic, we first look at the end of the peak cell rate burst. The peak cell rate phase stops as soon as the next cell will cause a leaky bucket overflow. Once the leaky bucket has been completely filled with the last cell we will have to wait $T_{s}$ time slots before the next cell can arrive. However, we also have to face the situation where the leaky bucket has been filled with the last cell in such a kind that there is remaining space for nearly one cell. But now, already in the next few time slots a new cell can fit into in the leaky bucket. This situation is shown in Figure 2 and it can be seen that the necessary buffer is larger than for greedy ON/OFF traffic. (Figure 2 shows an example for the superposition of $N=5$ WCT-B cell streams again with the GCRA parameter set $(T=2$, $\tau=0, T_{s}=8, \tau_{s}=23$ ). It is important to know that the larger buffer is only needed when the cell following the peak cell rate phase is emitted in a specific window of time after the last cell of the peak cell rate burst: The earliest time slot is the $T+1$ st time slot, the latest time slot is the $N-1$ st time slot after the last cell of the peak cell rate burst. Therefore, the maximum buffer that is required depends on whether the peak buffer occupation is reached at the end of the peak cell rate phase or at the arrival of the following cell:

$K_{B}=\max \left(K_{A}, K_{A}+N-\left[T+M B S\left(T_{s}-T\right)-\tau_{s}\right]\right)$

In total the WCT-B cell stream emits $M B S+1$ cells in each period. There- 

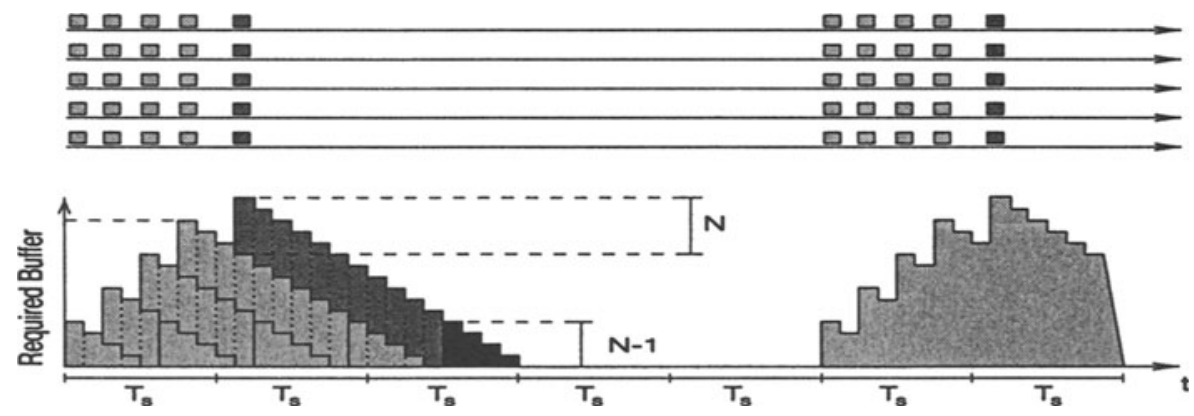

Figure 2 Synchronous WorstCaseTraffic-B Streams

from the period length is determined to be $\ell_{B}=\ell_{A}+T_{s}=\lceil M B S+1\rceil$. $T$, time slots

The maximum required buffer $K_{B}$ varies with the value of $\tau_{s}$. Therefore, the calculation of the mean number of free buffers $\kappa_{B}$ is not as simple as for the greedy ON/OFF traffic. In Figure 2 the light grey areas represent the buffers occupied by cells of the peak cell rate burst which already has been calculated in equation (2) while the darker areas show the additionally occupied buffer space. The graphical approach leads to:

$$
\begin{aligned}
\kappa_{B} & =\frac{1}{\ell_{B}} \cdot\left\{K_{B} \cdot \ell_{C}\right. \\
& -\frac{1}{2} M B S \cdot N[(N-1)+(N-T)(M B S-1)] \\
& +N \cdot\left[M B S\left(N-T_{s}\right)+\tau_{s}\right] \\
& \left.+\frac{1}{2} N(N-1)\right\} \text { cells/time slot }
\end{aligned}
$$

\section{TRAFFIC PARAMETERS FOR UPC AND CAC}

Both the connection admission algorithm and the usage parameter control need a set of parameters describing the statistical properties of the cell streams announced by the user. Ideally, the CAC would operate on exactly the same set of parameters advertised by a source to the UPC, because this set of parameters is enforced at the network boundary. Unfortunately, some of the most popular CAC methods were developed independent of the (later starting) discussion on UPC and therefore use other traffic parameters than those specified for the UPC. That's the case for our chosen pair of UPC and CAC as well. In general there is no unique transformation of the traffic parameters used by the two algorithms.

However, concentrating on the presented "worst case traffic" patterns, which have to be anticipated by a CAC to be on the safe side, the parameter transla- 
tion between UPC and CAC becomes unambiguous and therefore the question is well defined whether or not the CAC does its job properly under all traffic scenarios of "worst case" sources.

\subsection{Usage Parameter Control}

Following the interpretation in the Traffic Management Specification [1] there is a correspondence between the parameter set $\left(T, \tau(=0), T_{s}, \tau_{s}\right)$ and the peak cell rate (PCR), the sustainable cell rate (SCR), and the maximum burst size (MBS):

$$
\begin{aligned}
P C R & =1 / T \\
S R C & =1 / T_{s} \\
M B S & =\left\lfloor 1+\frac{\tau_{s}}{T_{s}-T}\right\rfloor
\end{aligned}
$$

where $\lfloor\bullet\rfloor$ means the integer part of $\bullet$.

\subsection{Connection Admission Control}

Among a multitude of CAC algorithms proposed $[4,5,6,7]$ the Equivalent Capacity Algorithm has both the virtue of broad acceptance and characterisation by three parameters, which brings it close to the three-parameter approach adopted for UPC in chapter 3.1:

Peak Bit Rate $R_{\text {peak }}$, Mean Bit Rate $\rho$, and Mean Burst Size $b$.

The latter two quantities are defined as mean values and it is not obvious in which way these means have to be determined: Can mean values be determined for connections lasting only a fraction of a second and can an application be aware of the duration of the connection (and the resulting averages) beforehand?

The way out of these conceptional problems is to consider each connection to be of "worst case" type - an assumption which might become reality with more sophisticated ATM communication boards, anyhow.

\subsection{Parameter Transformation}

The "worst case traffic" which we have presented is a periodic traffic. For this reason the calculation of the mean cell rate and the mean burst size is possible on the basis of a single period. Moreover, the mean cell rate coincides with the sustainable cell rate of the GCRA and, similarly, the mean burst size 
equals the maximum burst size and can be identified with this parameter of the GCRA setting.

\section{GCRA POLICER AND EQUIVALENT CAPACITY CAC}

Our traffic model is the following: Worst case cell streams are generated according to a set of $\left(T, T_{s}, \tau_{s}\right)$ so that no cells of these cell streams violate the traffic contract. From the parameter set $\left(T, T_{s}, \tau_{s}\right)$ the input parameters for the Equivalent Capacity CAC $\left(\rho, R_{P e a k}, b\right)$ are derived. A number of $N$ traffic streams will be multiplexed into a FIFO buffer with capacity $K$ and the cell loss is investigated where $N$ is the maximum number of connections that is accepted by the CAC algorithm. This means that only situations are investigated where the cell loss should not exceed the cell loss limit imposed on the CAC.

First we will present the synchronous superposition of cell streams where the burst phases of arriving cell streams all begin at the same time slot. Such a scenario must be seen as a worst case scenario when superposing cell streams. However, in general the cell streams will arrive with arbitrary phases. The impact of non-synchronised superposition of worst case cell streams is investigated in the second part of this section. Finally we extend our investigations from considering only one multiplexor to a simulation of worst case traffic in a 13 node ATM network model in section 4.3.

\subsection{Synchronised Superposition}

Using the Equivalent Capacity CAC that number of connections was determined for which the cell loss rate is estimated to stay below $\epsilon=10^{-5}$. The evaluation of equation (1) and (3) reveals to which degree this target is met. Figure 3 shows results for peak cell rates of $R_{P e a k}=0.1(T=10)$, $R_{\text {Peak }}=0.05(T=20)$, and combinations of $\tau_{s}$ and $T_{s}$ in a range from $20 \ldots 250$ and $20 \ldots 40$, respectively.

We distinguish three possible effects: Grey Areas indicate that the CAC Algorithm could protect the buffer from overload. Bright areas indicate combinations of $\tau_{s}$ and $T_{s}$, where the CAC still can deal worst case traffic type A properly but not worst case traffic type B. Areas plotted dark grey represent worst case traffic, where the CAC underestimates the impact on the multiplexer buffer for all types of worst case traffic. In these areas at least one cell is lost in every period of the cell stream.

The graphics show that the synchronous superposition of worst case cell streams causes huge problems to the Equivalent Capacity Algorithm. In contrast to what one might expect, the CAC performance is reduced furthermore, if the peak cell rate is reduced. This is because the Equivalent Capacity Al- 

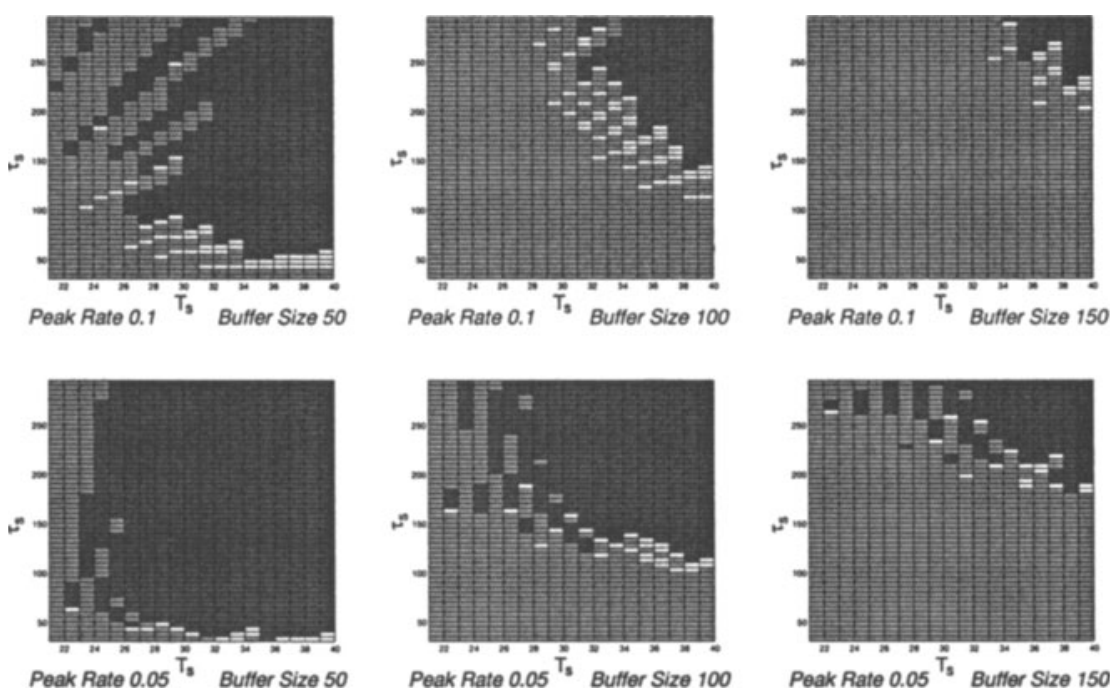

Figure 3 Performance of EquCap CAC: "worst case traffic" load with $10 \%$ and $5 \%$ peak cell rate

gorithm assumes a lower peak cell rate to be less demanding with respect to the necessary buffer size.

The second criterion to determine the "badness" of traffic streams is the amount of free buffer that is left for other traffic streams. We evaluate equations (2) and (4) for some example parameter sets which all lead to the burst size of 4 cells for WCT-A and 5 cells for WCT-B:

\begin{tabular}{ccccccccc}
\hline$\tau_{s}:$ & 23 & 22 & 21 & 20 & 19 & 18 & $\tau_{s}:$ & {$[18 \ldots 24[$} \\
\hline$K_{B}:$ & 15 & 14 & 13 & 13 & 13 & 13 & $K_{A}:$ & 13 \\
\hline$\kappa_{B}$ & 10.12 & 9.25 & 8.38 & 8.50 & 8.62 & 8.75 & $\kappa_{A}:$ & 8.94 \\
\hline
\end{tabular}

Table $1 K_{A}, \kappa_{A}, K_{B}$, and $\kappa_{B}$ for $T=2, \tau=0, T_{s}=8$, and $\tau_{s}=18 \ldots 23$

WCT-B is more demanding in terms of buffer required, although a higher buffer demand goes along with a greater average number of free buffers. In this context it is interesting to look to non worst case traffic streams. Based on $K=13$ as a buffer size to achieve comparable results we find for the mean number of free buffers for the synchronous superposition of $N=5$ constant cell rate streams $\left(T_{s}=8\right): \kappa_{C B R}=\frac{8 \cdot 13-10}{8}=11.75$ cells $/$ time slot. Using the mean buffer occupation for $\mathrm{N}-\mathrm{Geo} / \mathrm{D} / 1$ models for $N=5$ streams 
with geometrically distributed interarrival times $\kappa_{g e o}=13-1.024=11.98$ cells/time slot.

\subsection{Non-Synchronised Superposition of Cell Streams}

The synchronised superposition of cell streams represents the worst case traffic scenario, but it can be assumed that arbitrary phases of the cell streams will result in substantially lower cell loss rates. On the other hand, the number of phase constellations leading to unpredicted cell loss still may be very high. So far it is not easy to assess whether the overall cell loss rate will be lower or higher than the estimation of the CAC algorithm.

Due to the huge number of possible phase constellations an exhaustive analysis is not possible so far. E.g. a parameter set of Figure $3\left(T=20, \tau_{s}=\right.$ $\left.150, T_{s}=30\right)$ leads to $7.1 \cdot 10^{38}$ different phase constellations.

Although the problem dimension is very big, a tendency can be observed, when the phases are selected randomly. For different combinations of $\left(T, T_{s}, \tau_{s}\right)$ we have simulated the superposition of the corresponding number of cell streams with random phases, using a target cell loss rate of $\epsilon=10^{-4}$. Only the simulations with a 50 cell buffer have a target cell loss rate of $\epsilon=10^{-5}$ in order to be comparable with the results shown in Figure 3.

In Table 2 some results are collected. We have investigated three types of dependencies: The cell loss effects for different buffer sizes, different values of $\tau_{s}$, and different values of $T_{s}$. The respective parameter values are typed in bold letters.

In contrast to the synchronised superposition of the cell streams, the nonsynchronised superposition in general does not lead to higher cell loss than the target cell loss rate used by the CAC. Only for very small buffers the observed cell loss rate is slightly higher than aimed at.

\subsection{Extended ATM Network Model}

The simulation results presented here are obtained with the ATM network simulation software SABINE, operating on a 125 processor massive parallel computer. This simulation engine has been developed at the University of Technology Hamburg-Harburg $[8,9]$

Our realistic network model assumes an ATM backbone network for Germany consisting of 13 nodes interconnecting 13 larger cities. The number of links adapted to each ATM network node varies from node to node since the network topology follows geographic conditions. In our network the number of links per node varies between two and six. In addition to ATM links interconnecting the ATM network nodes, each node is supplied with an access link that is connected to an aggregate source model. The idea behind this type of 


\begin{tabular}{ccccccr}
\hline Buffer Size & $T$ & $T_{s}$ & $\tau_{s}$ & \# Connections & Total Load & Loss Rate \\
\hline $\mathbf{5}$ & 20 & 40 & 69 & 22 & 0.55 & $6.3 \cdot 10^{-4}$ \\
\hline $\mathbf{1 0}$ & 20 & 40 & 69 & 25 & 0.625 & $4.4 \cdot 10^{-7}$ \\
\hline $\mathbf{1 5}$ & 20 & 40 & 69 & 27 & 0.675 & $<10^{-7}$ \\
\hline 10 & 20 & 40 & $\mathbf{2 9 9}$ & 22 & 0.55 & $<10^{-7}$ \\
\hline 10 & 20 & 40 & $\mathbf{1 4 9}$ & 22 & 0.55 & $<10^{-7}$ \\
\hline 10 & 20 & 40 & $\mathbf{7 9}$ & 25 & 0.625 & $5.2 \cdot 10^{-7}$ \\
\hline 10 & 20 & 40 & $\mathbf{6 9}$ & 25 & 0.625 & $4.4 \cdot 10^{-7}$ \\
\hline 10 & 20 & 40 & $\mathbf{3 9}$ & 28 & 0.70 & $5.2 \cdot 10^{-6}$ \\
\hline 10 & 20 & $\mathbf{3 0}$ & 69 & 22 & 0.73 & $1.0 \cdot 10^{-6}$ \\
\hline 10 & 20 & $\mathbf{3 5}$ & 69 & 23 & 0.66 & $2.9 \cdot 10^{-7}$ \\
\hline 10 & 20 & $\mathbf{4 0}$ & 69 & 25 & 0.625 & $4.4 \cdot 10^{-7}$ \\
\hline 50 & 20 & $\mathbf{3 0}$ & $\mathbf{5 0}$ & 26 & 0.87 & $<10^{-7}$ \\
\hline 50 & 20 & $\mathbf{3 0}$ & $\mathbf{2 5 0}$ & 22 & 0.73 & $<10^{-7}$ \\
\hline 50 & 20 & $\mathbf{7 0}$ & $\mathbf{2 5 0}$ & 37 & 0.53 & $1.3 \cdot 10^{-6}$ \\
\hline 50 & 20 & $\mathbf{7 0}$ & $\mathbf{5 0}$ & 55 & 0.79 & $<10^{-7}$ \\
\hline 50 & 20 & $\mathbf{5 0}$ & $\mathbf{1 5 0}$ & 33 & 0.66 & $<10^{-7}$ \\
\hline
\end{tabular}

Table 2 Cell loss simulation for random phase superposition of WCT-B. Target cell loss rate is $10^{-5}$.

source modelling is that subscribers from a local network not are connected directly to the ATM backbone network, but are sharing one access link to the ATM backbone network.

The simulation results presented here are based on a number of subscribers who all are policed by the same set of GCRA parameters. Hence, they all produce the same worst case traffic. We have assumed the subscribers to be independent of each other, therefore no synchronised superposition of worst case traffic streams occur in this simulation. Due to the inhomogeneous network topology, some traffic streams only will pass two network nodes, while other traffic streams are routed through up to six network nodes. Furthermore, each traffic stream is multiplexed with a varying number of other traffic streams, which themselves already have been multiplexed in the same manner. All this leads to an unpredictably complex load scenario, which can be seen as a good approximation to a realistic ATM network situation. Compared to 
a simple Peak Rate Allocation in our example the admitted network load was substantially higher showing the efficiency of the Equivalent Capacity CAC. Depending on the burst size a factor between 3 and 11 could be obtained.

The simulation of this scenario yields that no cell loss could be observed at all. We interpret the result as follows: Due to the deterministic source behaviour in this scenario, statistical effects are reduced to random phases of the traffic streams. The Equivalent Capacity CAC obviously did not make too optimistic decisions and the critical situation of synchronous superposition of worst case streams did not occur within the simulation time. We have simulated the entire ATM network with a number of $10^{8}$ randomly selected phases of worst case traffic streams.

The simulation of a second scenario should consider statistical source behaviour. For this purpose we used statistical ON/OFF sources producing a basic traffic load. Onto this basic load a number of worst case traffic sources were loaded. It is expected that this number of worst case sources will lead to higher cell loss than the same number of statistical ON/OFF sources with the same load. The Equivalent Capacity CAC will not be able to distinguish between both types of traffic, so the interesting question is whether the same quality of service is observed or not. Simulation results reveal that the measured cell loss rate did not exceed the limit aimed at.

\section{CONCLUSION}

This paper was motivated by the question, whether the Equivalent Capacity Algorithm CAC and a GCRA based usage parameter control (UPC), in cooperation will protect the network from overload, even though, in general, they are based on different algorithms.

On the basis of the GCRA parameter set, we developed two types of worst case traffic that will pass the UPC but on the other hand will be most demanding for the buffer in a multiplexer.

In a first step, a fully synchronised superposition was assumed. Analytical expressions for the necessary buffer size at the input switch as a function of the GCRA parameters were derived. The analysis revealed, that the CAC algorithm was not able to protect the multiplexer against overload, and significant cell losses could be observed.

The synchronous superposition of cell streams is assumed to be very unlikely, although no one can guarantee that this situation will not happen. Hence, in a second step non synchronised cell streams were considered. An analytical approach for this scenario seems to be out of reach due to the huge number of possible phase constellations. Instead simulations were performed where the phases of the single cell streams were selected randomly. Compared with the synchronous superposition, the simulations of the non-synchronised cell streams yielded a much better performance of the CAC algorithm. The simulated cell loss rate for nearly all considered GCRA parameter sets was 
lower than the target cell loss rate used by the CAC. However, for small buffer sizes slightly higher cell loss rates were observed. This result was confirmed as the simulation was extended to a realistic network topology .

Our results show that if the source model is based on a two level leaky bucket with peak rate enforcement, Equivalent Capacity CAC and UPC can be reconciled on the basis of "worst case traffic" assumptions. Our experimental results show that in general the CAC will provide conservative but still sensible estimates of cell loss rates which allow loadings of the network in excess of $70 \%$.

Part of the work presented in this paper was supported by Deutsche Telekom AG, Darmstadt.

\section{REFERENCES}

[1] ATM Forum Traffic Management Specification 4.0, December 1995, ATM Forum/95-0013R9, pp63-68.

[2] B. T. Doshi: Deterministic Rule Based Traffic Descriptors for Broadband ISDN: Worst Case Behaviour and Connection Acceptance Control. Proceedings of the 14th International Teletraffic Congress, Antibes Juan-les-Pins, France, 6-10 June, 1994, pp 591-600.

[3] Roch Guerin, Hamid Ahmadi, Mahmoud Naghshineh: Equivalent capacity and its Application to Bandwidth Allocation in High Speed Networks. IEEE Journal on Selected Areas in Communications, Vol 9, No 7, September 1991, pp 968-981.

[4] Harry G. Perros, Khaled M. Elsayed: Call Admission Control Schemes: A Review. IEEE Communications Magazine, November 1996, pp. 82-91.

[5] Nikolas M. Mitrou, Kimon P. Kontovasilis, Hans Kröner, Villy Bæk Iversen: Statistical Multiplexing, Bandwidth Allocation Strategies and Connection Admission Control in ATM Networks. ETT, Vol 5, No 2, Mar.-Apr. 1994, pp. 33/161-47/175

[6] Gagan L. Choudhury, David M. Lucantoni, Ward Whitt: Squeezing the Most Out of ATM. IEEE Transactions on Communications, Vol 44, No 2, February 1996, pp. 203-217

[7] Ruth Kleinewillinghöfer-Kopp, Birgit Kaltenmorgen: Connection Acceptance Control in ATM Networks. Studie des Forschungsinstituts der DBP Telekom, Forschungsbereich 5 'Vermittlung und Netze', Juni 1991, also presented at: TIMS/ORSA Joint National Meeting, Nashville, Tennessee, USA, May 1991.

[8] K. Schmidt, U. Killat, F. Mayer-Lindenberg, P. Kraft: A Parallel ATM Network Simulator Proc. XV. International Switching Symposium ISS '95, April 1995, Berlin, Germany.

[9] K. Schmidt: Efficient ATM Network Simulation Proc. EUROSIM 1995 Conference, September 1995, Vienna, Austria. 